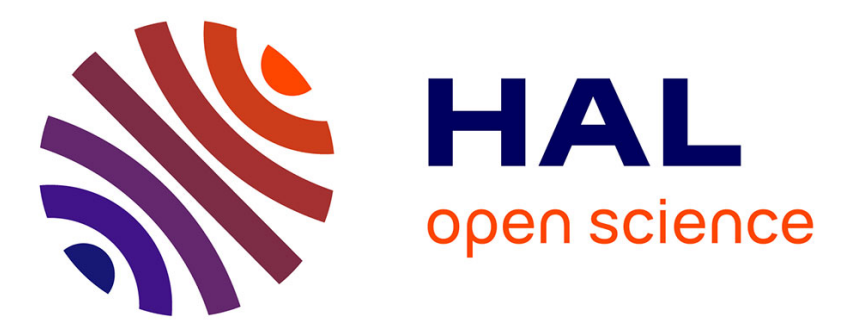

\title{
Zero focusing via competing nonlinearities in beta-barium-borate crystals
}

K. Krupa, F. Baronio, Matteo Conforti, Stefano Trillo, A. Tonello, V. Couderc

\section{To cite this version:}

K. Krupa, F. Baronio, Matteo Conforti, Stefano Trillo, A. Tonello, et al.. Zero focusing via competing nonlinearities in beta-barium-borate crystals. Optics Letters, 2014, 39 (4), pp.925-928. 10.1364/OL.39.000925 . hal-02389299

\section{HAL Id: hal-02389299 \\ https://hal.science/hal-02389299}

Submitted on 2 Dec 2019

HAL is a multi-disciplinary open access archive for the deposit and dissemination of scientific research documents, whether they are published or not. The documents may come from teaching and research institutions in France or abroad, or from public or private research centers.
L'archive ouverte pluridisciplinaire HAL, est destinée au dépôt et à la diffusion de documents scientifiques de niveau recherche, publiés ou non, émanant des établissements d'enseignement et de recherche français ou étrangers, des laboratoires publics ou privés. 
archives-ouvertes

\section{Zero focusing via competing nonlinearities in beta-barium-borate crystals}

K. Krupa, F. Baronio, M. Conforti, S. Trillo, A. Tonello, V. Couderc

\section{To cite this version:}

K. Krupa, F. Baronio, M. Conforti, S. Trillo, A. Tonello, et al.. Zero focusing via competing nonlinearities in beta-barium-borate crystals. Optics Letters, Optical Society of America, 2014, 39 (4), pp.925. 10.1364/OL.39.000925 . hal-02389299

\section{HAL Id: hal-02389299 \\ https://hal.archives-ouvertes.fr/hal-02389299}

Submitted on 2 Dec 2019

HAL is a multi-disciplinary open access archive for the deposit and dissemination of scientific research documents, whether they are published or not. The documents may come from teaching and research institutions in France or abroad, or from public or private research centers.
L'archive ouverte pluridisciplinaire HAL, est destinée au dépôt et à la diffusion de documents scientifiques de niveau recherche, publiés ou non, émanant des établissements d'enseignement et de recherche français ou étrangers, des laboratoires publics ou privés. 


\title{
Zero focusing via competing nonlinearities in BBO crystals
}

\author{
K. Krupa, ${ }^{1}$ F. Baronio,${ }^{2}$ M. Conforti, ${ }^{2}$ S. Trillo,${ }^{3}$ A. Tonello, ${ }^{1}$ V. Couderc,${ }^{1 *}$ \\ ${ }^{1}$ Institut Xlim, UMR 6172, CNRS, 123 avenue Albert Thomas, 87060 Limoges, France \\ ${ }^{2}$ Dipartimento di Ingegneria dell'Informazione, Università di Brescia, Via Branze 38, 25123 Brescia, Italy \\ ${ }^{3}$ Dipartimento di Ingegneria, Università di Ferrara, Via Saragat 1, 44122 Ferrara, Italy \\ ${ }^{*}$ Corresponding author: vincent.couderc@xlim.fr
}

Compiled November 4, 2013

We investigate nonlinear focusing behavior of light beams propagating in beta-barium-borate (BBO) crystals under mismatched second-harmonic generation. We clearly identify experimentally multiple self-focusing and defocusing regions against the orientation angle, and the condition where competing quadratic and cubic nonlinearities perfectly compensate each other (zero-focusing point).

OCIS codes: $190.2620,190.4223,190.3720$.

Introduction. Quadratic optical media are usually exploited for frequency conversion applications. However, the energy conversion process is always accompanied by nonlinear phase shifts of the interacting fields, as recognized long ago $[1,2]$. This concept remained nearly dormant until the beginning of the nineties when phase shifts produced in quadratic processes attracted a new surge of interest [3-6], also related to their exploitation for controlling optical signals in the spatial, temporal and spectral domains [7-17].

Any three-photon mixing process entail nonlinear phase shifts of all fields which, in the CW limit, are generally described by complicated periodic behavior with distance. However, in the regime of large phasemismatches, the phase shifts follow a Kerr-like behavior, being nearly proportional to distance and intensity [5]. This regime is also referred to as cascading limit [7] to recall the fact that the phase shifts accumulate through many repeated up- and down-conversion processes. Remarkably, such phase shift can be either self-induced [5] or cross-induced [6], while its sign depends on the sign of mismatch, and can be opposite for the different frequency components [10], thereby adding great flexibility in the applications.

Mostly exploited for its simplicity is the nonlinear phase shift of the fundamental frequency ( $\mathrm{FF}$ ) field occurring via second harmonic generation $(\mathrm{SHG})$ with no seed at second-harmonic ( $\mathrm{SH}$ ). In this configuration the cascaded phase shifts can be controlled through the wavevector mismatch by simply adjusting the orientation or the temperature of the crystal. Particularly interesting is the ability to generate negative phaseshifts which allows to access intriguing regimes (e.g. soliton propagation in normally dispersive bulk media and strong pulse compression and shaping without catastrophic collapse $[8,17]$ ), underlining the most innovative new possibilities [18-20].

However, in general, the net phase shift impressed on a light beam also contain a natural contribution from the cubic (Kerr) nonlinear index $n_{2}^{(3)}$ existing in any material and usually positive. Therefore, one might think about cascaded $\chi^{(2)}$ and $\chi^{(3)}$ processes as contributing to an overall effective nonlinear index as $n_{2}=n_{2}^{(2)}+n_{2}^{(3)}$ $[11,21]$. Since the cubic index contribution $n_{2}^{(3)}$ has negligible dependence on crystal orientation and temperature, it acts as a bias to the total nonlinear index. As a result, multiple self-focusing and self-defocusing regions can exist. For the typical case, when cubic phase shifts are positive, self-defocusing is expected to be possible within a finite range of mismatches (crystal orientations) on one side of phase-matching condition.

The aim of this Letter is to determine theoretically and experimentally such range for beta-bariumborate $(\mathrm{BBO})$ crystals. In particular, by observing the self-focusing and defocusing behavior of standard laser beams in BBO, we are able to clearly show the existence of a critical angle where the quadratic and cubic contributions mutually compensate for a wide range of input beam intensities, remarkably leading to a zero-focusing condition. To the best of our knowledge, compensation of this kind was achieved previously only by employing different crystals in back to back configuration [11]. Our experimental results agree well with theoretical analysis based on the cascading reduction of SHG equations, taking into account the Kerr coefficient of BBO.

Competing nonlinearities in BBO. We consider the propagation of an intense ordinarily polarized beam $E_{1}$ at FF $\omega=\omega_{0}$, together with its SH $\left(\omega=2 \omega_{0}\right)$, extraordinarily polarized beam $E_{2}$, generated through a type-I configuration. Since temporal effects over the time scale of the experiment are negligible, the following nonlinear coupled equations can be safely employed to describe SHG in this configuration [21-23]

$$
\begin{aligned}
i \frac{\partial E_{1}}{\partial z}+\frac{1}{2 k_{1}} \nabla_{\perp}^{2} E_{1} & +\chi_{2} E_{1}^{*} E_{2} e^{i \Delta k z} \\
& +\left(\chi_{31}\left|E_{1}\right|^{2}+\chi_{32}\left|E_{2}\right|^{2}\right) E_{1}=0 \\
i \frac{\partial E_{2}}{\partial z}+\frac{1}{2 k_{2}} \nabla_{\perp}^{2} E_{2} & +\chi_{2} E_{1}^{2} e^{-i \Delta k z} \\
& +\left(\chi_{33}\left|E_{1}\right|^{2}+\chi_{34}\left|E_{2}\right|^{2}\right) E_{2}=0
\end{aligned}
$$

where $E_{1,2}=E_{1,2}(x, y, z), \nabla_{\perp}^{2}=\partial^{2} / \partial x^{2}+\partial^{2} / \partial y^{2}, \chi_{2}$ 
and $\chi_{3 k}$ are proportional to the relevant elements of the quadratic and cubic susceptibility tensors, respectively $\left(\chi_{2}\right.$ refers to ooe three wave interaction, $\chi_{31}$ to oooo, $\chi_{32}$ to eeoo, $\chi_{33}$ to ooee, and $\chi_{34}$ to eeee four wave mixing interactions); $z$ is the propagation distance; $\Delta k=k_{2}-2 k_{1}=2\left[n_{e}\left(2 \omega_{0} ; \theta\right)-n_{o}\left(\omega_{0}\right)\right] \omega / c$ is the wavenumber mismatch, $n_{o}\left(\omega_{0}\right)$ and $n_{e}\left(2 \omega_{0} ; \theta\right)$ being the ordinary and extraordinary refractive index at $\mathrm{FF}$ and $\mathrm{SH}$, while $\theta$ is the angle of the propagation direction with respect to the optical axis of the crystal.

In Tables 1 and 2, we report the effective nonlinearities for the crystals of class $3 m$ to which BBO belongs, as a function of rotation angles $(\theta, \phi)$ by specifying the relative type of interaction. For instance, ooe $(o+o \rightarrow e)$ indicates the upconversion of two ordinarily polarized photons that generate an extraordinarily polarized photon at SH (for further details see Refs. [24, 25]).

\begin{tabular}{lc} 
Coefficient Expression & Interaction \\
\hline$d_{0}=-3 d_{31} \cos ^{2} \theta \sin \theta-d_{22} \cos ^{3} \theta \sin 3 \phi$ & eee \\
$d_{1}=-d_{22} \cos 3 \phi \cos ^{2} \theta$ & eeo, oeo, oee \\
$d_{2}=-d_{31} \sin \theta+d_{22} \cos \theta \sin 3 \phi$ & ooe, eoo, oeo \\
$d_{3}=d_{22} \cos 3 \phi$ & ooo
\end{tabular}

Table 1. Effective quadratic nonlinear coefficients. $d_{22}=$ $2.2 \mathrm{pm} / \mathrm{V}, d_{31}=-0.04 \mathrm{pm} / \mathrm{V}$.

\begin{tabular}{lrl} 
Coefficient Expression & Interaction \\
\hline$c_{0}=$ & $c_{11} \cos ^{4} \theta+c_{33} \sin ^{4} \theta+\frac{3}{2} c_{16} \sin ^{2} 2 \theta$ & eeee \\
& $-4 c_{10} \sin 3 \phi \sin \theta \cos ^{3} \theta$ & \\
$c_{1}=$ & $\frac{3}{2} c_{10} \cos 3 \phi \sin 2 \theta \cos \theta$ & eeoe, eeeo \\
$c_{2}=$ & $-\frac{1}{3} c_{11} \cos ^{2} \theta+c_{16} \sin ^{2} \theta$ & ooee,eeoo \\
& $+c_{10} \sin 2 \theta \sin 3 \phi$ & \\
$c_{3}=c_{10} \cos 3 \phi \sin \theta$ & oooe, ooeo \\
$c_{4}=c_{11}$ & oooo
\end{tabular}

Table 2. Effective cubic nonlinear coefficients. $c_{11}=5.6$. $10^{-22} \mathrm{~m}^{2} / \mathrm{V}^{2}, c_{10}=-0.24 \cdot 10^{-22} \mathrm{~m}^{2} / \mathrm{V}^{2}, c_{16}=-1.4$. $10^{-22} \mathrm{~m}^{2} / \mathrm{V}^{2}[24]$.

In the cascading regime (high $|\Delta k|$ ), the leading order equation that can be derived from Eqs. (1) is the following nonlinear Schrödinger equation that governs the evolution the ordinary FF beam $E_{1}[21,26]$ :

$$
i \frac{\partial E_{1}}{\partial z}+\frac{1}{2 k_{1}} \nabla_{\perp}^{2} E_{1}+\left(\gamma_{2}+\gamma_{3}\right)\left|E_{1}\right|^{2} E_{1}=0
$$

where cascaded quadratic and cubic Kerr nonlinearities are expressed as $\gamma_{2}=-\left(\frac{\omega_{0} d_{e f f}}{n_{0} c}\right)^{2} \frac{1}{\Delta k}\left[\mathrm{~m} / V^{2}\right]$ and $\gamma_{3}=$ $\frac{3}{8} \frac{\omega_{0} c_{e f f}}{n_{0} c}\left[m / V^{2}\right]$, where $d_{e f f}=d_{1}$ and $c_{e f f}=c_{4}$ are the effective nonlinear coefficients from Tables 1, 2 .

Figure 1 shows the effective nonlinear index $\gamma=$ $\gamma_{2}+\gamma_{3}$, resulting from cascading-quadratic $\gamma_{2}$, and cubic $\gamma_{3}$ contributions, as function of the angle $\theta$, at fixed $\phi=90^{\circ}$. Multiple self-focusing and defocusing regions can be identified. In particular, in the region of positive mismatches $\left(\gamma_{2}<0\right)$, a zero-focusing point is identified at $\theta_{c}=18.4^{\circ}$, where the quadratic and cubic contributions exactly balance $\left(\gamma_{2}=-\gamma_{3}\right)$. While for angles $\theta<\theta_{c}$ the mismatch increases and hence the Kerr effect prevails leading to self-focusing, for increasing angles $\theta>\theta_{c}$ the mismatch decreases and leads cascading to dominate thus resulting into self-defocusing, until for $\theta$ approaching the phase-matching angle $\theta=22.9^{\circ}$, the cascading picture breaks down due to increased efficiency of SHG (shaded region in Fig. 1). For angles greater than the perfect phase-matching angle, $\gamma_{2}$ and $\gamma_{3}$ have the same sign, leading to enhanced focusing.

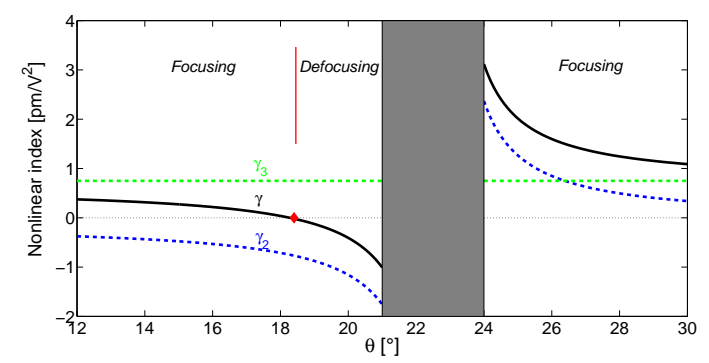

Fig. 1. (Color online) Total effective cubic nonlinearity $\gamma$, resulting from both quadratic $\gamma_{2}$ and cubic $\gamma_{3}$ contributions, plotted versus the angle $\theta$. The shaded domain identifies the region where cascading breaks down and SHG enters the regime of efficient conversion.

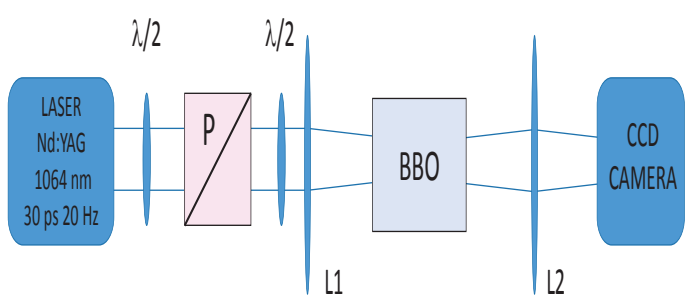

Fig. 2. (Color online) Experimental setup. Nd:YAG laser pump delivering $30 \mathrm{ps}$ pulses, at $1064 \mathrm{~nm}, 20 \mathrm{~Hz}$ repetition rate; $\mathrm{L}$ lenses; $\lambda / 2$ half wave plates; $\mathrm{P}$ polarizer; quadratic uniaxial BBO crystal; CCD camera.

Experimental results. A sketch of our experimental setup is reported in Fig. 2. We employ a sample of BBO crystal with a square aperture of $6 \times 6 \mathrm{~mm}$ and a length of $20 \mathrm{~mm}$. The crystal is cut for frequency doubling at $1064 \mathrm{~nm}$ under type-I (ooe) phase-matching, at the angles $\theta=22.9^{\circ}$ and $\phi=90^{\circ}$ to the principal axes. The crystal faces are coated for antireflection at $1064 \mathrm{~nm}$. The crystal is pumped using a Q-switched, mode-locked $\mathrm{Nd}$ :YAG laser which delivers $30 \mathrm{ps}$ pulses at $1064 \mathrm{~nm}$ at a repetition rate of $20 \mathrm{~Hz}$, with energy up to $1.23 \mathrm{~mJ}$. 
A polarizer in between half-wave plates are used to adjust the energy and the polarization of the pump pulses. The pump light is collimated using a lens of $140 \mathrm{~mm}$ focal length. The average beam diameter at the input is around $140 \mu \mathrm{m}$ FWHM. The spatial beam patterns at the output of the crystal are imaged, with magnification $G=4.6$, onto a CCD camera and analyzed. Under such experimental conditions, we characterize the spatial dynamics of an ordinary $\mathrm{FF}$ beam $E_{1}$ at the output of the $\mathrm{BBO}$ crystal, at different crystal orientation angles and input energies.

In Fig. 3 we summarize the dependence of the beam diameter (FWHM) at the output of the BBO crystal versus crystal orientation angle $\theta$, for different input energies. First notice that diffraction has by itself negligible impact due to the large beam size (see curve relative to the linear limit in Fig. 3), while it becomes obviously effective, in the nonlinear regime, to transform the selfinduced spatial phase modulation into beam narrowing (self-focusing) or broadening (self-defocusing). The data indicate, as expected from theory, the existence of different nonlinear focusing and defocusing regions. In particular in the region of negative phase mismatches $\left(\theta>24^{\circ}\right.$ in Fig. 3), the cascading effect always adds up to the Kerr coefficient leading to self-focusing (beam narrowing). Conversely for positive mismatches we observe opposite behavior across the critical angle $\theta_{c}=18.6^{\circ}$, at which the beam travels nearly undistorted due to mutual balance of Kerr and cascading nonlinear index. While for $\theta<18.6^{\circ}$, the $\mathrm{FF}$ beam self-focuses due to dominant positive Kerr index, for $18.6^{\circ}<\theta<22.5^{\circ}$ the cascading nonlinear index overwhelms the Kerr effect causing the beam to defocus. Remarkably, while increasing the input energy (intensity) results into an enhanced nonlinear effect, the point of zero-focusing remains the same with good accuracy regardless of the beam intensity. In order

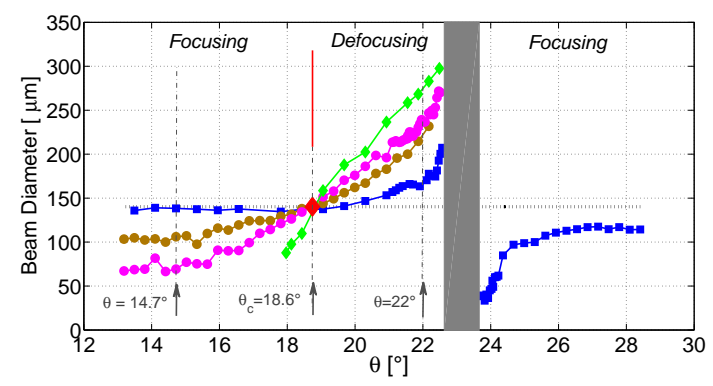

Fig. 3. (Color online) Beam diameter (FWHM of intensity) at FF measured at BBO crystal output vs. crystal rotation angle $\theta$, for different energy of the pulse: linear regime (dotted line); $0.08 \mathrm{~mJ}$ (blue squares); $0.34 \mathrm{~mJ}$ (gold circles); $0.54 \mathrm{~mJ}$ (magenta circles); $1.23 \mathrm{~mJ}$ (green diamonds).

to show that the beams undergo self-focusing or defocusing without substantial distortion, we also display in Fig. 4 the beam profiles obtained for fixed input energy ( 0.54 $\mathrm{mJ}$ ) for three characteristic values of the angle $\theta$ across the critical value $\theta_{c}$ (vertical lines in Fig. 3 ). In particular the defocusing behavior in Fig. 4(a,d), obtained for $\theta=22^{\circ}$ above the critical value $\theta_{c}$, is contrasted with the focusing behavior in Fig. 4(c,f), obtained below the critical value $\theta=14.7^{\circ}<\theta_{c}$, while Fig. 4(b,e) corresponds to the critical angle that give rise to the undistorted propagation.
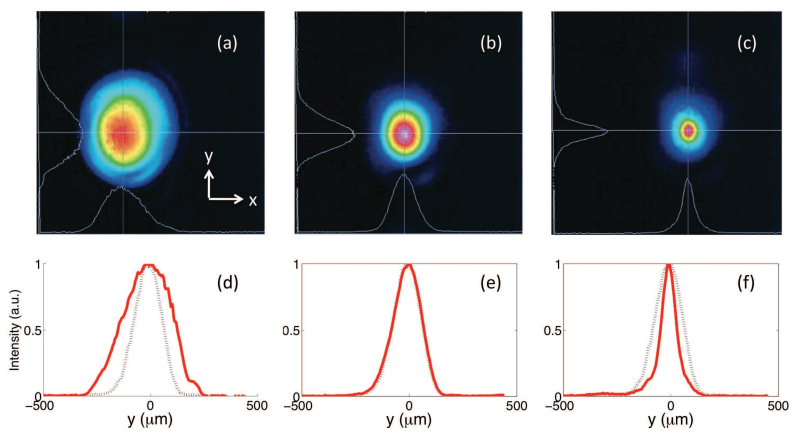

Fig. 4. (Color online) (a-c) measured 2D output beam shapes and (d-f) corresponding beam profiles vs. $y(x=$ 0 section), compared with the input. (a,d) $\theta=22^{\circ}$; (b,e) $\theta=\theta_{c}=18.6^{\circ} ;(\mathrm{c}, \mathrm{f}) \theta=14.7^{\circ}$. Here the input energy is $0.54 \mathrm{~mJ}$.

Conclusions. We have considered the effect of nonlinear phase shifts impressed on light beams during propagation in $\mathrm{BBO}$ crystals, showing evidence for competing quadratic and cubic contributions. We have identified theoretically and have demonstrated experimentally the existence of multiple overall self-focusing and defocusing regions, and the existence for a critical value of orientation which yields a compensation of cascading and cubic contributions.

Acknowledgments F.B., M.C. and S.T. acknowledge funding by the Italian Ministry of University and Research (MIUR), under grant PRIN 2009P3K72Z. K.K., A.T. and V.C. acknowledge the financial support provided by OSEO within the frame of the project VERTICAL.

\section{References}

1. J.A. Armstrong, N. Bloembergen, J. Ducuing and P.S. Pershan, Phys. Rev. 127, 1918 (1962).

2. J.M.R. Thomas and J.P.E. Taran, Opt. Commun. 4, 329 (1972).

3. N.R. Belashenkov, S.V. Gagarskii and M.V. Inochkin, Opt. Spectrosc. 66, 806 (1989).

4. H. J. Bakker, P. C. M. Planken, L. Kuipers, and A. Lagendijk, Phys. Rev. A 42, 4085 (1990).

5. R. DeSalvo, D. J. Hagan, M. Sheik-Bahae, G. Stegeman, E. W. Van Stryland, and H. Vanherzeele, Opt. Lett. 17, 28 (1992). 
6. D. C. Hutchings, J. S. Aitchison, and C. N. Ironside, Opt. Lett. 18, 793 (1993).

7. G. I. Stegeman, D. J. Hagan and L. Torner, Opt. Quantum Electron. 28, 1691 (1996).

8. X. Liu, L. Qian, and F. W. Wise, Opt. Lett. 24, 1881 (1999).

9. K. Gallo, P. Baldi, M. De Micheli, D. B. Ostrowsky, and G. Assanto, Opt. Lett. 25, 966 (2000).

10. P. Di Trapani, A. Bramati, S. Minardi, W. Chinaglia, C. Conti, S. Trillo, J. Kilius, and G. Valiulis, Phys. Rev. Lett. 87, 183902 (2001).

11. K. Beckwitt, F. Wise, L. Qian, L. A. Walker II, and E. Canto-Said, Opt. Lett. 26, 1696 (2001).

12. F. Baronio, C. De Angelis, P. Pioger, V. Couderc, A. Barthelemy, Y. Min, V. Quiring, and W. Sohler, Opt. Lett. 28, 2348 (2003).

13. J. Prawiharjo, K. Gallo, N. Broderick, and D. J. Richardson, J. Opt. Soc. Am. B 22, 1985 (2005).

14. F. Baronio, C. De Angelis, M. Marangoni, C. Manzoni, R. Ramponi, and G. Cerullo, Opt. Express 14, 4774 (2006)

15. X. Zeng, S. Ashihara, Z. Wang, T. Wang, Y. Chen, and M. Cha, Opt. Express 17, 16877 (2009).

16. M. Marangoni, G. Sanna, Brida, M. Conforti, G. Cirmi, C. Manzoni, F. Baronio, and P. Bassi, App. Phys. Lett. 93, 021107 (2008).

17. F. W. Wise and J. Moses, in Self-focusing: Past and Present, R. W. Boyd, S. G. Lukishova and Y. R. Shen, eds., (Springer, 2009), pp. 481-506.

18. M. Bache, O. Bang, B. B. Zhou, J. Moses, and F. W. Wise, Phys. Rev. A 82, 063806 (2010).

19. B. B. Zhou, A. Chong, F.W. Wise, and M. Bache, Phys. Rev. Lett. 109, 04392 (2012).

20. M. Conforti, F. Baronio and S. Trillo, Opt. Lett. 37, 1082 (2012).

21. S. Trillo, A. V. Buryak, and Y. S. Kivshar, Opt. Commun. 122, 200 (1996)

22. A. V. Buryak, Y. S. Kivshar and S. Trillo, Opt. Lett. 19, 1961 (1995).

23. M. Bache, O. Bang, J. Moses and F. Wise, Opt. Lett. 32, 2490 (2007)

24. M. Bache, H. Guo, B. Zhou, and X. Zeng, Opt. Mat. Express 3, 357 (2013).

25. M. Conforti and F. Baronio, J. Opt. Soc. Am. B 30, 1041 (2013).

26. C.R. Menyuk, R. Schiek, and L. Torner, J. Opt. Soc. Am. B 11, 2434 (1994). 


\section{Informational Fifth Page}

\section{References}

1. J.A. Armstrong, N. Bloembergen, J. Ducuing and P.S. Pershan, "Interactions between light waves in a nonlinear dielectric," Phys. Rev. 127, 1918 (1962).

2. J.M.R. Thomas and J.P.E. Taran, "Pulse distortions in mismatched second harmonic generation," Opt. Commun. 4, 329 (1972).

3. N.R. Belashenkov, S.V. Gagarskii, and M.V. Inochkin, "On the nonlinear light refraction under the 2ndharmonic generation," Opt. Spectrosc. 66, 806 (1989).

4. H. J. Bakker, P. C. M. Planken, L. Kuipers, and A. Lagendijk, "All-optical switching based on nondegenerate phase shifts from a cascaded second-order nonlinearity", Phys. Rev. A 42, 4085 (1990).

5. R. DeSalvo, D. J. Hagan, M. Sheik-Bahae, G. Stegeman, E. W. Van Stryland, and H. Vanherzeele, "Selffocusing and self-defocusing by cascaded 2nd-order effects in KTP," Opt. Lett. 17, 28 (1992).

6. D. C. Hutchings, J. S. Aitchison, and C. N. Ironside, "All-optical switching based on nondegenerate phase shifts from a cascaded second-order nonlinearity", Opt. Lett. 18, 793 (1993).

7. G. I. Stegeman, D. J. Hagan and L. Torner, " $\chi^{(2)}$ cascading phenomena and their applications to all-optical signal processing, mode-locking, pulse compression and solitons," Opt. Quantum Electron. 28, 1691 (1996).

8. X. Liu, L. Qian, and F. W. Wise, "High-energy pulse compression by use of negative phase shifts produced by the cascade $\chi^{(2)}: \chi^{(2)}$ nonlinearity," Opt. Lett. 24, 1881 (1999).

9. K. Gallo, P. Baldi, M. De Micheli, D. B. Ostrowsky, and G. Assanto, "Cascading phase shift and multivalued response in counterpropagating frequency-nondegenerate parametric amplifiers," Opt. Lett. 25, 966 (2000).

10. P. Di Trapani P., Bramati A., Minardi S., Chinaglia W., Conti C., Trillo S., Kilius J. and Valiulis G., "Focusing versus defocusing nonlinearities in self-trapping due to parametric frequency conversion," Phys. Rev. Lett. 87, 183902 (2001).

11. K. Beckwitt, F. Wise, L. Qian, L. A. Walker II, and E. Canto-Said, "Compensation for self-focusing by use of cascade quadratic nonlinearity," Opt. Lett. 26, 1696 (2001).

12. F. Baronio, C. De Angelis, P. Pioger, V. Couderc, A. Barthelemy, Y. Min, V. Quiring, and W. Sohler, "Soliton emission at a phase-mismatch boundary in a quadratic nonlinear film waveguide," Opt. Lett. 28, 2348 (2003).

13. J. Prawiharjo, K. Gallo, N. Broderick, and D. J. Richardson, "Frequency-resolved optical gating in the $1.55-\mu \mathrm{m}$ band via cascaded chi(2) processes," J. Opt. Soc. Am. B 22, 1985 (2005).

14. F. Baronio, C. De Angelis, M. Marangoni, C. Manzoni, R. Ramponi, and G. Cerullo, "Spectral shift of femtosecond pulses in nonlinear quadratic PPSLT Crystals," Opt. Express 14, 4774 (2006).

15. X. Zeng, S. Ashihara, Z. Wang, T. Wang, Y. Chen, and M. Cha, "Excitation of two-colored temporal solitons in a segmented quasi-phase-matching structure," Opt. Express 17, 16877 (2009).

16. M. Marangoni, G. Sanna, Brida, M. Conforti, G. Cirmi,
C. Manzoni, F. Baronio, and P. Bassi, "Observation of spectral drift in engineered quadratic nonlinear media," C. De Angelis, and G. Cerullo, App. Phys. Lett. 93, 021107 (2008).

17. F. W. Wise and J. Moses, "Self-focusing and self-defocusing of femtosecond pulses with cascaded quadratic nonlinearities," in Self-focusing: Past and Present, R. W. Boyd, S. G. Lukishova and Y. R. Shen, eds., (Springer, 2008), pp. 481-506.

18. M. Bache, O. Bang, B. B. Zhou, J. Moses, and F. W. Wise, "Optical Cherenkov radiation in ultrafast cascaded second-harmonic generation," Phys. Rev. A 82, 063806 (2010).

19. B. B. Zhou, A. Chong, F.W. Wise, and M. Bache, "Ultrafast and Octave-Spanning Optical Nonlinearities from Strongly Phase-Mismatched Quadratic Interactions" Phys. Rev. Lett. 109, 04392 (2012).

20. M. Conforti, F. Baronio and S. Trillo, "Dispersive shock waves in phase-mismatched second harmonic generation" Opt. Lett. 37, 1082 (2012).

21. S. Trillo, A. V. Buryak, and Y. S. Kivshar, "Modulational instability and optical solitons due to competition of $\chi^{(2)}$ and $\chi^{(3)}$ nonlinearities," Opt. Commun. 122, 200 (1996).

22. A. V. Buryak, Y. S. Kivshar and S. Trillo, "Optical solitons supported by competing nonlinearities," Opt.Lett. 19, 1961 (1995).

23. M. Bache, O. Bang, J. Moses and F. Wise, "Nonlocal explanation of stationary and nonstationary regimes in cascaded soliton pulse compression," Opt. Lett. 32, 2490 (2007).

24. M. Bache, H. Guo, B. Zhou, and X. Zeng, "The anisotropic Kerr nonlinear refractive index of the betabarium borate $\beta-\mathrm{BaB}_{2} \mathrm{O}_{4}$ nonlinear crystal," Opt. Mat. Express 3, 357 (2013).

25. M. Conforti and F. Baronio, "Extreme high-intensity and ultrabroadband interactions in anisotropic $\beta-$ $\mathrm{BaB}_{2} \mathrm{O}_{4}$ crystals" J. Opt. Soc. Am. B 30, 1041 (2013).

26. C.R. Menyuk, R. Schiek, and L. Torner, "Solitary waves due to $\chi^{(2)}: \chi^{(2)}$ cascading" J. Opt. Soc. Am. B 11, 2434 (1994). 